\title{
Seitenwechsel erweitert den Horizont und schafft Vertrauen
}

\author{
Medizin im Managementkontext verstehen - ein neues Fortbildungsangebot fördert \\ den vertieften Dialog zwischen den «Kulturen» und zentralen Entscheidungsträgern.
}

Lukas Zemp

Generalsekretär/

Geschäftsstellenleiter

Zusätzliche Informationen: www.fhnw.ch/wirtschaft/ weiterbildung/cas oder www.sgim.ch/veranstaltung

Korrespondenz: Lukas Zemp Schweizerische Gesellschaft für Allgemeine Innere Medizin (SGIM)

Solothurnerstrasse 68

CH-4008 Basel

Tel. 0612359335

info[at]sgim.ch

\begin{abstract}
Managerinnen und Manager in Spitälern und Gesundheitsorganisationen brauchen zunehmend medizinische Kenntnisse, um die medizinischen Abläufe in ihrer Komplexität zu verstehen sowie für einen vertieften Dialog mit den medizinischen Partnern. Dieses spezifische Wissen fehlt ihnen aber oft. Diese Lücke hilft das neue Fortbildungsangebot «CAS-Medicine for Managers» zu schliessen. Das ab April 2014 geplante neue Fortbildungsangebot legt im Interesse der Patienten zusätzlich besonderes Gewicht auf Inhalte und Kompetenzen im Kontext der Allgemeinen Inneren Medizin (AIM) und der Hausarztmedizin.
\end{abstract}

In den letzten Jahren ist eine Tendenz festzustellen, dass Medizinalberufe zunehmend mit betriebswirtschaftlichen Themen konfrontiert werden. Die Ökonomisierung der Medizin ist als Schlagwort auch medial omnipräsent. Dabei geht es im Wesentlichen darum, dass Kernaufgaben der medizinischen Leistungserbringer im Spital oder der Praxis mit Hilfe betriebswirtschaftlichen Denkens und Handelns im Sinne von problemlösungsorientiertem Anwenden betriebswirtschaftlicher Werkzeuge ergänzt werden. In der Unterstützungs- und Versorgungsebene eines Spitals oder einer Gemeinschaftspraxis arbeiten überwiegend Fachpersonen mit betriebswirtschaftlichem, kaufmännischem oder medizin-technischem Hintergrund. Diese Personengruppen haben selten einen biologischen oder medizinischen Hintergrund. Während es zahlreiche betriebswirtschaftliche Diplom- und Nachdiplomausbildungen auf universitärer Ebene gibt, ist eine Weiterbildungslücke im medizinischen Bereich für Nicht-Mediziner festzustellen. Ein Weiterbildungsangebot auf Fachhochschulniveau in Medizin hätte zum Ziel, die Kommunikation zwischen Personen in strategischen Versorgungseinheiten (z. B. Logistik, Finanzen, Patientenadministration) von Spitälern, Praxisgemeinschafen, Pharmaindustrie und medizin-technischen Firmen auf der einen Seite und den mit medizinischen Kernaufgaben betrauten Ärzt(inn)en auf der anderen Seite zu verbessern.

\section{Seitenwechsel und «Brückenbauer-Funktion»}

Geplant ist, ein kurzes, aber intensives medizinisches Fortbildungsprogramm für wichtige Entscheidungsträger im Gesundheitswesen aus Verwaltung, Institutionen, Industrie und Handel zu erarbeiten: den «Certificate of Advanced Studies (CAS) - Medicine for Managers» der Fachhochschule Nordwestschweiz (FHNW) in Zusammenarbeit mit der Klinik und Poliklinik für
Innere Medizin des UniversitätsSpitals Zürich (USZ) sowie der Schweizerischen Gesellschaft für Allgemeine Innere Medizin (SGIM). Im Sinne eines «Seitenwechsels» sollen die verschiedenen Entscheidungsträger für medizinische Fragen und Prozesse und insbesondere für die Anliegen der Allgemeinen Inneren Medizin und der Hausarztmedizin sensibilisiert werden. Der Pilotkurs, der für April 2014 in deutscher Sprache geplant ist, möchte eine Brücke bauen, um das Verständnis zwischen den «Kulturen» sowie Menschen mit und ohne medizinischen Kernaufgaben zu fördern. Der Kurs bietet die Möglichkeit, konzentriert Kenntnisse der Pathologie, der Physiologie und des medizinischen Managements zu erwerben und bei Meetings mit Medizinern diese Kenntnisse einzusetzen. Dieses Grundlagenwissen der Medizin soll dabei mit spezifischen Lerninhalten des medizinischen Curriculums (TARMED, DRG, Evidence based Medicine) verknüpft werden. Wer das Pflichtenheft des Arztes inhaltlich nachvollziehen kann, ist besser in der Lage, spezifische Managementaufgaben auf medizinische Betreuungsinhalte abzustimmen. Zielgruppen des neuen Angebots sind in erster Linie das mittlere und höhere Kader mit betriebswirtschaftlichem oder kaufmännischem Hintergrund aus Spitälern, KMU, Bank- und Versicherungssektor sowie Pharmaund Medizin-Technikbranche.

\section{Kursangebot im Interesse der Patienten}

Die Idee zu diesem Projekt ist beim ersten Managementfortbildungsangebots «CAS - Management of Medical Units» 2013 entstanden. Der Initiant und CoLeiter des neuen Fortbildungsangebots, PD Dr. med. Jens P. Hellermann, Leitender Arzt Innere Medizin/ Kardiologie, Spitalregion Rheintal Werdenberg Sarganserland, war Teilnehmer der ersten Managementfortbildung 2013 von SGIM und FHNW. Die SGIM verspricht sich vom neuen Kursangebot für NichtMedziner eine Verbesserung und Intensivierung des partnerschaftlichen Dialogs zwischen den verschiedenen Schlüsselpersonen im Gesundheitswesen und zum Nutzen der Patienten und des Schweizer Gesundheitswesens. Zusätzlich begrüsst die SGIM die besondere Fokussierung des neuen Kursangebots auf die Anliegen der Allgemeinen Inneren Medizin (AIM) sowie der Hausarztmedizin im Rahmen der geplanten Fortbildungsmodule. 